\title{
More Wavelengths From Thorium Lamps
}

\author{
William F. Meggers and Robert W. Stanley*
}

(December 13, 1964)

\begin{abstract}
For the purpose of supplementing or superseding the spectroscopic secondary standards of wavelength derived since 1910 from an iron arc, Meggers and Stanley, in 1958, reported the first interferometric determinations of wavelengths emitted by a thorium lamp. Those determinations were restricted to 222 intense radiations of thorium with wavelengths ranging from $3288.7356 \AA$ to $6991.5839 \AA$. Now the same, and additional, interference spectrograms have been measured to provide improved wavelengths for 510 radiations ranging in wavelength from $3269.6089 \AA$ to $7020.504 \AA$. The present list includes many lines of lower intensity than those previously published and fills most of the large intervals in our first report. The accuracy in relative wavelength values of 163 classified thorium lines is tested by the combination principle which indicates that the average error is less than 1 part in 20 to 40 million. Similar measurements of wavelengths emitted by iron-halide lamps have errors that are 3 to 5 times greater.
\end{abstract}

\section{Introduction}

After forty years of intermittent effort to extend and improve the international standards of wavelengths used in spectroscopy, Meggers $[1]^{1}$ suggested, in 1955, that secondary standards, superior in sharpness and distribution, could be obtained by replacing the standard iron arc with a modern thorium lamp as a light source. Such improvement was demonstrated by Meggers and Stanley [2] who first measured the wavelengths of 222 intense radiations emitted by a thorium-iodide lamp. Those wavelengths extended from $3288.7356 \AA$ to 6991.5839 $\AA$ in vacuum; they were measured interferometrically relative to wavelengths emitted by a Meggers mercury 198 lamp. In the meantime, those measurements have found favor among all spectroscopists who specialize in making accurate descriptions of complex spectra, especially of chemical elements whose atoms are more massive than those of iron. Moreover, those spectroscopists have suggested that more wavelengths of thorium radiations be accurately measured to increase the range of intensity and decrease the average interval, thus removing handicaps on account of overexposure and/or expanded dispersion in modern photographic spectrograms. Consequently, the same, and additional, interference spectrograms have been measured to provide inproved values of wavelengths for 510 thorium radiations (including 222 reported earlier) ranging in value from $3263.6089 \AA$ to $7020.504 \AA$ in vacuum. The purpose of this paper is to present a new list of thorium wavelengths including many lines of lower intensity than those previously published [2] and also to reduce the average interval between

\footnotetext{
*Present address, Purdue University, Lafayette, Ind.
}

1 Figures in brackets indicate the literature references at the end of this paper. standards. Because of the increase in the number of carefully measured wavelengths, the combination principle is applied as an internal test of the accuracy in relative value of a considerable fraction of them.

\section{Experiments}

In our first paper [2], full details concerning light sources, interferometers, spectrograph, measurements, and computations were given for the first determinations of thorium wavelengths from FabryPerot etalons. Briefly, the thorium radiations were emitted by a small electrodeless lamp containing a few milligrams of thorium iodide and several torr of helium. The wavelengths of selected thorium lines were measured relative to adopted standards emitted by a similar lamp containing $1 \mathrm{mg}$ of mercury-198 and 3 torr of argon. Both lamps were excited by magnetrons generating $2450 \mathrm{Mc} / \mathrm{s}$. One lamp was imaged in the other and both simultaneously illuminated a Fabry-Perot etalon interferometer to avoid errors usually entailed by alternate exposures to different light sources.

The enclosed and evacuated Fabry-Perot interferometer consisted of two flat plates of crystalline quartz whose facing surfaces were coated with thin aluminum films and separated by invar etalons of 25,40 , or $50 \mathrm{~mm}$ length. The 25 and $40 \mathrm{~mm}$ spacers were relied upon to insure the correct integral order of interference for any given line, assuming that available values of the wavelength obtained from diffraction-grating spectrograms had no errors greater than $\pm 0.02 \AA$.

The interference patterns (Haidinger fringes) were photographed with a concave grating of 22 feet radius in a (stigmatic) Wadsworth mounting. The spectral range $4400 \AA$ to $7000 \AA$ was photographed in the first-order spectrum with a slit width of $0.30 \mathrm{~mm}$ and plate factor of $5 \AA / \mathrm{mm}$. Because 
of a greater density of thorium lines in the unltraviolet, the region from $3250 \AA$ to $4500 \AA$ was recorded in the second-order grating spectrum with a plate factor of $2.5 \AA / \mathrm{mm}$ and slit width of $0.15 \mathrm{~mm}$. In each wavelength range, several spectrograms were made with different times of exposure, and with slightly different spacing of the interferometer plates to alter the interference configurations or fractional orders for all spectral lines. The green $(5462.2705 \AA)$ and violet $\left(4047.7144 \AA\right.$ ) lines of $\mathrm{Hg}^{198}$ were chosen as standards, respectively, for the longand short-wave interference spectrograms described above. The wavelengths of $\mathrm{Hg}^{198}$ radiations used for the calibration of etalons were previously measured [3] relative to red radiation $(6438.4696 \AA)$ of cadmium, the primary standard of wavelength prior to 1960 .

In our first report [2] , the fractional order at the center of an interference pattern was determined from measurements of five interference fringes for each line and least-squares calculations according to the formula

$$
\epsilon=\frac{2\left(3 \mathrm{D}_{1}+2 \mathrm{D}_{2}^{2}+\mathrm{D}_{3}^{2}-\mathrm{D}_{5}^{2}\right)}{-2 \mathrm{D}_{1}^{2}-\mathrm{D}_{2}^{2}+\mathrm{D}_{4}^{2}+2 \mathrm{D}_{5}^{2}}
$$

first discussed by Rolt and Barrell [4]. After taking squares from a table, all computations were by mental arithmetic except the final quotient which was obtained from a desk calculator. About 12,000 interference fringes were thus used to derive the wavelengths of 222 intense radiations in thorium spectra, 172 from Th $\mathrm{I}$ and 50 from Th II.

The results reported in the present paper were obtained by Meggers from spectrograms made by Stanley in 1956 and described jointly in 1958 [2]. In addition, several spectrograms that were overexposed, and, therefore, unsuitable for measurement of the stronger lines, were salvaged by dissolving the dense background in solutions of sodium thiosulphate and potassium ferricyanide, thereby making them ideal for measurement of the weaker lines.

In this series of measurements, the diameters of six interference circles (Haidinger fringes) were determined from readings of the distances between twelve fringes for each line. In most cases, two readings were made on each fringe and the mean was recorded. Since the fringes on 510 thorium lines and several mercury 198 lines were measured on two to six spectrograms, the average being four, it appears that more than 40,000 readings were made. The recorded readings were transferred to IBM cards in a punch machine and transmitted to an electronic computer that calculated wavelengths after determining the fractional and integral orders of interference for each line in a given interferometer.

The fractional order, $\epsilon$, at the center of an interference pattern was calculated from 6 diameters according to the formula:

$$
\epsilon=\frac{5}{3}\left(\frac{11 \mathrm{D}_{1}^{2}+8 \mathrm{D}_{2}^{2}+5 \mathrm{D}_{3}^{2}+2 \mathrm{D}_{4}^{2}-\mathrm{D}_{5}^{2}-4 \mathrm{D}_{6}^{2}}{-5 \mathrm{D}_{1}^{2}-3 \mathrm{D}_{2}^{2}-\mathrm{D}_{3}^{2}+\mathrm{D}_{4}^{2}+3 \mathrm{D}_{5}^{2}+5 \mathrm{D}_{6}^{2}}\right)
$$

The final calculations of wavelength, $\lambda$, were made from the relation $\lambda=2 t /(p+\epsilon)$, where $t$ is the separation of the interferometer plates, $p$ is the integral order, and $\epsilon$ the fractional order of interference at the center of a pattern. In this series of measurements, the wavelengths of 510 thorium radiations were determined relative to $\mathrm{Hg}^{198}$ wavelengths $(5462.2706 \AA, 4359.5625 \AA$, and $4047.7147 \AA$ ) which in turn were measured by Kaufman [5] in terms of the new primary standard of length, 6057.80211 $\AA$, radiated by $\mathrm{Kr}^{86}$.

\section{Results}

Our latest measurements of vacuum wavelengths for 510 radiations from thorium lamps are presented in table 1 . The vacuum values in column 1 were converted to appropriate values in standard air, column 2, by using the Table of Wavenumbers of Coleman, Bozman, and Meggers [6]. The estimated relative intensities, column 3 , and assignment to spectrum, Th I or Th II, are quoted from a New Description of Thorium Spectra by Zalubas [7]. Table 1 contains data for 510 radiations, 395 from Th I and 115 from Th II. Wavelength values given to 8 figures have estimated errors less than 0.0005 $\AA$, 34 given to 7 figures are included to suggest that they are suitable for further refinement.

Although the $\mathrm{Kr}^{86}$ wavelength (6057.80211 $\AA$ in vacuum) was derived from the $\mathrm{Cd}$ wavelength (6438.46960 $\AA$, in air), the wavelengths of identical $\mathrm{Hg}^{198}$ lamps (3 torr Ar) measured relative to the former are slightly greater $(0.0001$ to $0.0003 \AA)$ than those derived from the latter. Consequently, the wavelengths of 222 thorium radiations reported in our first paper [2] have now increased by 0.0001 $\AA$ on the average. We are unable to explain the source of this small discrepancy. Fortunately for spectroscopic use as standards, the absolute values of thorium wavelengths are less important than their relative values for calibrating spectrograms.

Because the number of independent determinations of each thorium wavelength is, in general, too small to yield a true probable error, we have extensively applied the combination principle as a rigid test of the accuracy of measured wavelengths in relative value. Two radiations resulting from combinations of a highly excited atomic (or ionic) level with two low-energy levels will exhibit in their wavenumbers the energy difference of the low levels. Any other high levels that combine with the same low levels will exhibit the same difference within the accuracy of measurement. In table 2, we present typical examples of 21 low-energy differences, 20 involving wavenumber differences between designated lowenergy levels for Th I [8], and 1 for ThII [9]. The total number of line pairs in table 2 is 111 but, since some radiations participate two or three times in pairing with others, only 163 different lines are involved. However, these are randomly distributed throughout the list and comprise nearly 63 percent of the classified Th I wavelengths given to 8 figures. 
We may expect, therefore, that the remainder will satisfy these tests when other wavelengths are measured and the present partial analyses of these spectra are greatly extended.

The critical test of our thorium wavelengths is shown in the last two columns of table 2, where recurring wavenumber differences and their deviations from the mean are listed.

The deviations of the measured level separations from the mean range from 0 to $\pm 4 \mathrm{~m} \mathrm{~K}$ (milliKayser $=10^{-3} \mathrm{~cm}^{-1}$ ). The average deviation of all tested pairs of lines is $\pm 0.0012 \mathrm{~K}$, and if we assume the errors are shared equally by all lines, the individual average error is $0.0006 \mathrm{~K}$, or one part in 20 to 40 million, depending on the spectral region. These tests indicate that our thorium wavelengths qualify as Class A standards defined as having accuracy of $0.001 \mathrm{~K}$ according to a suggestion by Littlefield [10]. Calculated probable errors, as well as atomic energy intervals, for similar measurements of wavelengths from iron-halide lamps [11] indicate that the relative values are uncertain by 1 part in 7 or 8 million.

Recently, additional measurements of thorium wavelengths have been reported by Littlefield and Wood [12], by Davison, Giacchetti, and Stanley [13], and by Giacchetti, Gellarado, Garavaglia, Gonzalez, Valero, and Zakowicz [14]. The first [12] used a hollow-cathode light source and reflectionechelon interferometer to measure the wavelengths of 484 thorium radiations ranging from 2566.3615 $\AA$ to $9050.7361 \AA$; the results have been reported in the Transactions of the I A U but no further experimental details have been published. The others, [13, 14], used electrodeless thorium lamps and Fabry-Perot interferometers similar to ours. Davison et al. [13] published the wavelengths of 68 radiations ranging from $2651.3722 \AA$ to 3394.9671 $\AA$, and Giacchetti et al. [14] gave 129 values from $2687.9304 \AA$ to $4596.7088 \AA$. Unfortunately, none of the above-mentioned observers tested their results by means of the combination principle; there are many serious discrepancies between values reported by two or more observers and it will be important to seek the source of the larger differences.

The senior author gladly and gratefully acknowledges the assistance of Mrs. John B. Peterson who transferred written readings to punched cards, and thanks Victor Kaufman for presenting these data to an electronic computer that produced wavelengths by using a code previously prepared by Karl G. Kessler.

Washington, D.C., November 2, 1964
TABLE 1. Wavelengths of thorium lines

\begin{tabular}{|c|c|c|c|}
\hline$\lambda$ vac & $\lambda$ air & $\begin{array}{l}\text { Relative } \\
\text { intensity }\end{array}$ & Spectrum \\
\hline \begin{tabular}{l}
\multicolumn{1}{c}{$\AA$} \\
3263.6089 \\
3272.9913 \\
3274.8374 \\
3276.011 \\
3286.6984
\end{tabular} & \begin{tabular}{l}
\multicolumn{1}{c}{$\AA$} \\
3262.6682 \\
3272.0482 \\
3273.8938 \\
3275.067 \\
3285.7518
\end{tabular} & $\begin{array}{r}3000 \\
800 \\
600 \\
400 \\
900\end{array}$ & $\begin{array}{l}\text { II } \\
\text { I } \\
\text { II } \\
\text { II } \\
\text { I }\end{array}$ \\
\hline $\begin{array}{l}3288.7358 \\
3292.6868 \\
3293.4688 \\
3302.207 \\
3305.1895\end{array}$ & $\begin{array}{l}3287.7887 \\
3291.7387 \\
3292.5205 \\
3301.256 \\
3304.2382\end{array}$ & $\begin{array}{r}1600 \\
3000 \\
3000 \\
600 \\
3000\end{array}$ & $\begin{array}{l}\text { II } \\
\text { II } \\
\text { II } \\
\text { II } \\
\text { I }\end{array}$ \\
\hline $\begin{array}{l}3310.3178 \\
3314.022 \\
3321.4317 \\
3325.7088 \\
3326.0767\end{array}$ & $\begin{array}{l}3309.3652 \\
3313.068 \\
3320.4763 \\
3324.7523 \\
3325.1201\end{array}$ & $\begin{array}{r}800 \\
400 \\
400 \\
2000 \\
3000\end{array}$ & $\begin{array}{l}\text { I } \\
\text { I } \\
\text { I } \\
\text { II } \\
\text { II }\end{array}$ \\
\hline $\begin{array}{l}3331.4345 \\
3334.0972 \\
3338.8297 \\
3349.7310 \\
3352.1912\end{array}$ & $\begin{array}{l}3330.4765 \\
3333.1285 \\
3337.8698 \\
3348.768 \\
3351.2279\end{array}$ & $\begin{array}{l}1800 \\
1000 \\
2000 \\
3000 \\
2500\end{array}$ & $\begin{array}{c}\text { I } \\
\text { I } \\
\text { II } \\
\text { I } \\
\text { II }\end{array}$ \\
\hline $\begin{array}{l}3359.5667 \\
3368.7860 \\
3375.9440 \\
3379.5433 \\
3381.8300\end{array}$ & $\begin{array}{l}3358.6015 \\
3367.8185 \\
3374.9747 \\
3378.5730 \\
3380.8592\end{array}$ & $\begin{array}{r}2000 \\
1200 \\
1600 \\
400 \\
900\end{array}$ & $\begin{array}{c}\text { II } \\
\text { II } \\
\text { I } \\
\text { II } \\
\text { I }\end{array}$ \\
\hline $\begin{array}{l}3386.5031 \\
3393.0082 \\
3397.7023 \\
3399.5201 \\
3406.5348\end{array}$ & $\begin{array}{l}3385.5311 \\
3392.0345 \\
3396.7274 \\
3398.5447 \\
3405.5576\end{array}$ & $\begin{array}{r}800 \\
6000 \\
1400 \\
1600 \\
1400\end{array}$ & $\begin{array}{c}\text { II } \\
\text { II } \\
\text { I } \\
\text { I } \\
\text { I }\end{array}$ \\
\hline $\begin{array}{l}3413.9919 \\
3422.1909 \\
3424.9714 \\
3434.9827 \\
3438.2923\end{array}$ & $\begin{array}{l}3413.0128 \\
3421.2097 \\
3423.9895 \\
3433.9983 \\
3437.3070\end{array}$ & $\begin{array}{l}1800 \\
2500 \\
1600 \\
3000 \\
2500\end{array}$ & $\begin{array}{c}\text { I } \\
\text { I } \\
1 \\
\text { II } \\
\text { I }\end{array}$ \\
\hline $\begin{array}{l}3443.5653 \\
3452.6909 \\
3458.0588 \\
3463.8419 \\
3469.2125\end{array}$ & $\begin{array}{l}3442.5787 \\
3451.7019 \\
3457.0685 \\
3462.8501 \\
3468.2193\end{array}$ & $\begin{array}{r}800 \\
900 \\
800 \\
2000 \\
2000\end{array}$ & $\begin{array}{l}\text { I } \\
\text { I } \\
\text { I } \\
\text { II } \\
\text { II }\end{array}$ \\
\hline $\begin{array}{l}3480.1683 \\
3487.5248 \\
3494.5176 \\
3499.6217 \\
3504.7880\end{array}$ & $\begin{array}{l}3479.1723 \\
3486.5269 \\
3493.5179 \\
3498.6207 \\
3503.7857\end{array}$ & $\begin{array}{r}800 \\
3500 \\
2000 \\
900 \\
500\end{array}$ & $\begin{array}{l}\text { II } \\
\text { II } \\
\text { II } \\
\text { I } \\
\text { I }\end{array}$ \\
\hline $\begin{array}{l}3512.1610 \\
3519.4096 \\
3522.0660 \\
3531.5236 \\
3532.4595\end{array}$ & $\begin{array}{l}3511.1568 \\
3518.4035 \\
3521.0592 \\
3530.5144 \\
3531.4501\end{array}$ & $\begin{array}{r}1000 \\
1000 \\
500 \\
500 \\
1000\end{array}$ & $\begin{array}{c}\text { I } \\
\text { I } \\
\text { I } \\
\text { II } \\
\text { I }\end{array}$ \\
\hline $\begin{array}{l}3540.3335 \\
3540.5985 \\
3545.0303 \\
3550.6096 \\
3552.4160\end{array}$ & $\begin{array}{l}3539.3220 \\
3539.5870 \\
3544.0176 \\
3549.5955 \\
3551.4014\end{array}$ & $\begin{array}{r}800 \\
4000 \\
1500 \\
1200 \\
1000\end{array}$ & $\begin{array}{l}\text { II } \\
\text { II } \\
\text { I } \\
\text { I } \\
\text { I }\end{array}$ \\
\hline $\begin{array}{l}3556.0284 \\
3560.4658 \\
3564.3930 \\
3568.2824 \\
3573.4119\end{array}$ & $\begin{array}{l}3555.0129 \\
3559.4491 \\
3563.3753 \\
3567.2637 \\
3572.3919\end{array}$ & $\begin{array}{r}1500 \\
2500 \\
800 \\
1200 \\
1000\end{array}$ & $\begin{array}{c}\text { I } \\
\text { II } \\
\text { I } \\
\text { I } \\
\text { II }\end{array}$ \\
\hline
\end{tabular}


TABLE 1. Wavelengths of thorium lines-Continued

\begin{tabular}{|c|c|c|c|}
\hline$\lambda \mathrm{vac}$ & $\lambda$ air & $\begin{array}{l}\text { Relative } \\
\text { intensity }\end{array}$ & Spectrum \\
\hline $\begin{array}{c}\stackrel{̊}{\AA} \\
3577.5785 \\
3585.1986 \\
3592.4771 \\
3593.8042 \\
3599.1464\end{array}$ & $\begin{array}{c}\AA \\
3576.5574 \\
3584.1756 \\
3591.4522 \\
3592.7790 \\
3598.1198\end{array}$ & $\begin{array}{r}1000 \\
800 \\
1000 \\
2000 \\
2000\end{array}$ & $\begin{array}{l}\text { I } \\
\text { I } \\
\text { I } \\
\text { I } \\
\text { I }\end{array}$ \\
\hline $\begin{array}{l}3605.711 \\
3606.222 \\
3609.4067 \\
3613.4576 \\
3616.1634\end{array}$ & $\begin{array}{l}3604.683 \\
3605.194 \\
3608.3774 \\
3612.4273 \\
3615.1324\end{array}$ & $\begin{array}{r}800 \\
500 \\
1200 \\
1400 \\
2000\end{array}$ & $\begin{array}{l}\text { I } \\
\text { I } \\
\text { I } \\
\text { I } \\
\text { II }\end{array}$ \\
\hline $\begin{array}{l}3623.8281 \\
3626.6612 \\
3633.8656 \\
3636.9796 \\
3643.2867\end{array}$ & $\begin{array}{l}3622.7951 \\
3625.6275 \\
3632.8300 \\
3635.9432 \\
3642.2487\end{array}$ & $\begin{array}{r}800 \\
500 \\
1000 \\
1900 \\
2200\end{array}$ & $\begin{array}{c}\text { I } \\
\text { II } \\
\text { I } \\
\text { I } \\
\text { I }\end{array}$ \\
\hline $\begin{array}{l}3650.7746 \\
3657.7354 \\
3662.6653 \\
3669.1843 \\
3671.0138\end{array}$ & $\begin{array}{l}3649.7346 \\
3656.6936 \\
3661.6223 \\
3668.1396 \\
3669.9686\end{array}$ & $\begin{array}{r}1200 \\
1000 \\
500 \\
1000 \\
750\end{array}$ & $\begin{array}{c}\text { I } \\
\text { I } \\
\text { II } \\
\text { I } \\
\text { I }\end{array}$ \\
\hline $\begin{array}{l}3672.5853 \\
3676.6136 \\
3683.5345 \\
3691.6742 \\
3693.6172\end{array}$ & $\begin{array}{l}3671.5397 \\
3675.5670 \\
3682.4861 \\
3690.6237 \\
3692.5662\end{array}$ & $\begin{array}{r}700 \\
1000 \\
1000 \\
1000 \\
1200\end{array}$ & $\begin{array}{r}\text { I } \\
\text { II } \\
\text { I } \\
\text { I } \\
\text { I }\end{array}$ \\
\hline $\begin{array}{l}3699.1584 \\
3702.0314 \\
3707.8219 \\
3712.3597 \\
3720.4927\end{array}$ & $\begin{array}{l}3698.1059 \\
3700.9782 \\
3706.7672 \\
3711.3038 \\
3719.4347\end{array}$ & $\begin{array}{r}1300 \\
300 \\
2400 \\
600 \\
3000\end{array}$ & $\begin{array}{l}\text { I } \\
\text { I } \\
\text { I } \\
\text { II } \\
\text { I }\end{array}$ \\
\hline $\begin{array}{l}3728.9626 \\
3734.0467 \\
3734.7340 \\
3742.2464 \\
3743.9873\end{array}$ & $\begin{array}{l}3727.9024 \\
3732.9852 \\
3733.6723 \\
3741.1828 \\
3742.9232\end{array}$ & $\begin{array}{r}800 \\
500 \\
500 \\
3000 \\
1100\end{array}$ & $\begin{array}{c}\text { I } \\
\text { I } \\
\text { I } \\
\text { II } \\
\text { I }\end{array}$ \\
\hline $\begin{array}{l}3748.6040 \\
3753.6352 \\
3758.7620 \\
3764.0030 \\
3771.1268\end{array}$ & $\begin{array}{l}3747.5387 \\
3752.5686 \\
3757.6941 \\
3762.9337 \\
3770.0557\end{array}$ & $\begin{array}{r}900 \\
3500 \\
1000 \\
1200 \\
1300\end{array}$ & $\begin{array}{c}\text { II } \\
\text { II } \\
\text { I } \\
\text { I } \\
\text { I }\end{array}$ \\
\hline $\begin{array}{l}3772.4419 \\
3777.3435 \\
3782.0398 \\
3786.6751 \\
3790.2449\end{array}$ & $\begin{array}{l}3771.3704 \\
3776.2708 \\
3780.9659 \\
3785.5999 \\
3789.1688\end{array}$ & $\begin{array}{r}1500 \\
600 \\
350 \\
1000 \\
1500\end{array}$ & $\begin{array}{c}\text { I } \\
\text { I } \\
\text { I } \\
\text { II } \\
\text { I }\end{array}$ \\
\hline $\begin{array}{l}3796.4632 \\
3804.1547 \\
3808.9554 \\
3814.1499 \\
3819.7690\end{array}$ & $\begin{array}{l}3795.3855 \\
3803.0750 \\
3807.8745 \\
3813.0676 \\
3818.6853\end{array}$ & $\begin{array}{r}500 \\
4000 \\
1200 \\
1200 \\
500\end{array}$ & $\begin{array}{l}\text { II } \\
\text { I } \\
\text { II } \\
\text { II } \\
\text { I }\end{array}$ \\
\hline $\begin{array}{l}3829.4708 \\
3831.8604 \\
3838.9638 \\
3840.7835 \\
3843.0499\end{array}$ & $\begin{array}{l}3828.3845 \\
3830.7735 \\
3837.8751 \\
3839.6943 \\
3841.9601\end{array}$ & $\begin{array}{r}3200 \\
800 \\
2000 \\
2500 \\
1200\end{array}$ & $\begin{array}{l}\text { I } \\
\text { I } \\
\text { I } \\
\text { I } \\
\text { II }\end{array}$ \\
\hline $\begin{array}{l}3853.2278 \\
3855.6037 \\
3860.9341 \\
3864.5011 \\
3870.7604\end{array}$ & $\begin{array}{l}3852.1354 \\
3854.5106 \\
3859.8396 \\
3863.4057 \\
3869.6634\end{array}$ & $\begin{array}{r}600 \\
1200 \\
500 \\
1200 \\
600\end{array}$ & $\begin{array}{l}\text { I } \\
\text { II } \\
\text { II } \\
\text { II } \\
\text { I }\end{array}$ \\
\hline
\end{tabular}

TABLE 1. Wavelengths of thorium lines-Continued

\begin{tabular}{|c|c|c|c|}
\hline$\lambda$ vac & $\lambda$ air & $\begin{array}{l}\text { Relative } \\
\text { intensity }\end{array}$ & Spectrum \\
\hline $\begin{array}{c}\AA \\
3875.9602 \\
3876.4723 \\
3880.7435 \\
3885.9251 \\
3896.5229\end{array}$ & $\begin{array}{c}\AA \\
3874.8618 \\
3875.3738 \\
3879.6439 \\
3884.8241 \\
3895.4192\end{array}$ & $\begin{array}{r}1800 \\
500 \\
1000 \\
400 \\
400\end{array}$ & $\begin{array}{c}\text { I } \\
\text { I } \\
\text { I } \\
\text { II } \\
\text { I }\end{array}$ \\
\hline $\begin{array}{l}3904.2082 \\
3905.169 \\
3906.2923 \\
3913.0171 \\
3917.5266\end{array}$ & $\begin{array}{l}3903.1025 \\
3904.063 \\
3905.1860 \\
3911.9091 \\
3916.4174\end{array}$ & $\begin{array}{r}1500 \\
1000 \\
1500 \\
600 \\
500\end{array}$ & $\begin{array}{c}\text { I } \\
\text { II } \\
\text { II } \\
\text { I } \\
\text { I }\end{array}$ \\
\hline $\begin{array}{l}3920.1336 \\
3924.9106 \\
3926.2046 \\
3930.7818 \\
3934.0244\end{array}$ & $\begin{array}{l}3919.0239 \\
3923.7995 \\
3925.0932 \\
3929.6692 \\
3932.9109\end{array}$ & $\begin{array}{r}800 \\
400 \\
1000 \\
2500 \\
1400\end{array}$ & $\begin{array}{c}\text { I } \\
\text { I } \\
\text { I } \\
\text { II } \\
\text { I }\end{array}$ \\
\hline $\begin{array}{l}3938.1548 \\
3944.5112 \\
3950.0814 \\
3953.8796 \\
3960.4204\end{array}$ & $\begin{array}{l}3937.0402 \\
3943.3950 \\
3948.9637 \\
3952.7609 \\
3959.3000\end{array}$ & $\begin{array}{r}400 \\
400 \\
1000 \\
500 \\
1000\end{array}$ & $\begin{array}{l}\text { II } \\
\text { II } \\
\text { II } \\
\text { I } \\
\text { I }\end{array}$ \\
\hline $\begin{array}{l}3968.5146 \\
3973.2783 \\
3974.3200 \\
3981.2151 \\
3988.3509\end{array}$ & $\begin{array}{l}3967.3921 \\
3972.1546 \\
3973.1960 \\
3980.0893 \\
3987.2232\end{array}$ & $\begin{array}{r}2000 \\
1400 \\
1100 \\
1100 \\
600\end{array}$ & $\begin{array}{l}\text { I } \\
\text { I } \\
\text { I } \\
\text { I } \\
\text { I }\end{array}$ \\
\hline $\begin{array}{l}3991.6206 \\
3995.6788 \\
4002.1893 \\
4003.0253 \\
4009.3433\end{array}$ & $\begin{array}{l}3990.4921 \\
3994.5492 \\
4001.0580 \\
4001.8938 \\
4008.2102\end{array}$ & $\begin{array}{r}800 \\
1200 \\
800 \\
150 \\
1600\end{array}$ & $\begin{array}{l}\text { I } \\
\text { I } \\
\text { I } \\
\text { I } \\
\text { I }\end{array}$ \\
\hline $\begin{array}{l}4010.190 \\
4013.6295 \\
4019.2344 \\
4020.2649 \\
4028.1470\end{array}$ & $\begin{array}{l}4009.057 \\
4012.4952 \\
4018.0987 \\
4019.1289 \\
4027.0089\end{array}$ & $\begin{array}{r}1600 \\
2000 \\
400 \\
1200 \\
1000\end{array}$ & $\begin{array}{l}\text { I } \\
\text { I } \\
\text { I } \\
\text { II } \\
\text { I }\end{array}$ \\
\hline
\end{tabular}

4031. 4314

4031. 9815

4037. 1879

4037. 7056

4044. 5369

4052. 0315

4054. 6727

4060. 3994

4064. 5547

4065. 4793

4068. 5994

4070. 3508

4070. 6098

4073. 7787

4076. 6536

4082. 5200

4087. 6742

4089. 8815

4090. 2920

4995. 9029

4098. 9042

4101. 4985

4109. 5792

4110. 4832

4113. 9150
4030. 2925

4030,8424

4036. 0475

4036. 5650

4043. 3946

4050. 8872

4053. 5277

4059. 2529

4063. 4071

4064. 3315

4067. 4508

4069. 2017

4069. 4606

4072. 6287

4075. 5029

4081. 3677

4086. 5206

4088. 7273

4089. 1377

4094. 7471

4097. 7476

4100. 3413

4108. 4198

4109. 3236

4112. 7545

\begin{tabular}{r|r}
200 & I \\
1800 & I \\
1800 & I \\
400 & II \\
800 & I \\
& \\
800 & I \\
1000 & I \\
1000 & I \\
800 & II \\
400 & I \\
& \\
400 & I \\
750 & II \\
400 & I \\
400 & I \\
400 & I \\
800 & \\
1600 & I \\
400 & II \\
400 & I \\
1600 & I \\
800 & II \\
1100 & I \\
800 & I \\
400 & II \\
700 & I \\
& I
\end{tabular}


TABLE 1. Wavelengths of thorium lines-Continued

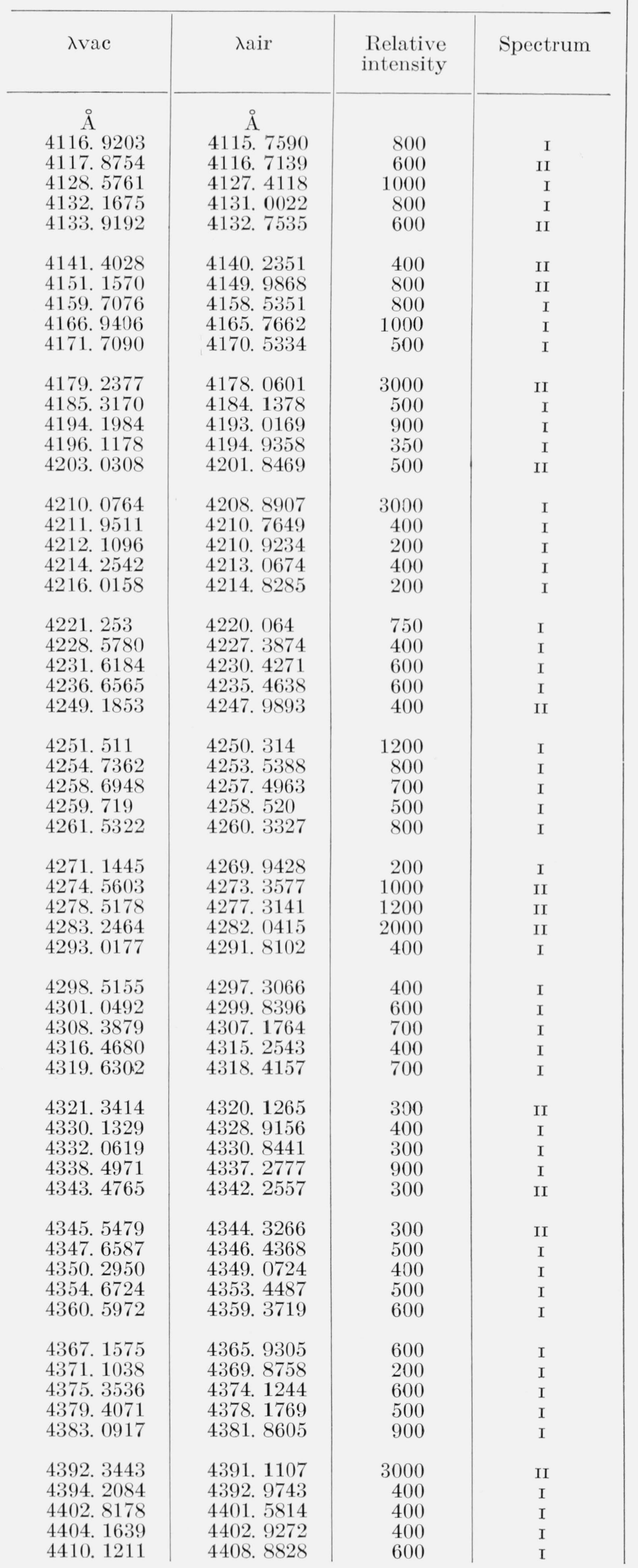

TABLE 1. Wavelengths of thorium lines-Continued

\begin{tabular}{|c|c|c|c|}
\hline$\lambda$ vac & 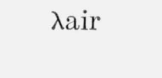 & $\begin{array}{l}\text { Relative } \\
\text { intensity }\end{array}$ & Spectrum \\
\hline$\AA$ & $\AA$ & & \\
\hline 4415.7260 & 4414.4862 & 400 & I \\
\hline 4423.2900 & 4422.0482 & 350 & I \\
\hline 4434.2077 & 4432.9640 & 600 & II \\
\hline 4440.370 & 4439.124 & 400 & II \\
\hline 4442.114 & 4440.867 & 400 & II \\
\hline 4446.5562 & 4445.3083 & 300 & I \\
\hline 4447.1495 & 4445.9014 & 200 & I \\
\hline 4453.8148 & 4452.5650 & 200 & I \\
\hline 4459. 2531 & 4458.0018 & 600 & I \\
\hline 4462.4933 & 4461.2412 & 600 & I \\
\hline 4466.5940 & 4465.3408 & 300 & II \\
\hline 4470.7800 & 4469.5257 & 400 & I \\
\hline 4483.4270 & 4482.1694 & 300 & I \\
\hline 4488.1563 & 4486.8974 & 200 & I \\
\hline 4494.5943 & 4493.3337 & 1200 & I \\
\hline
\end{tabular}

4500.2022

4501.2459

4506. 4799

4511.7910

4516. 3845

4522,489

4531.5895

4536. 5265

4542. 2720

4547. 1900

4557. 0903

4562. 6266

4572. 2528

4589. 7124

4593. 953

4596. 7082

4604. 461

4622.4565

4633.0585

4641. 344

4652.857

4664.5080

4669.4785

4671. 2916

4674. 9693

4687. 5060

4696. 3520

4705. 3063

4713. 7713

4719. 9363

4730. 4507

4741. 8552

4753. 7432

4762. 4415

4767. 9332

4779. 6300

4790. 7256

4809. 4775

4810. 9583

4824. 2027

4828. 0490 4832. 4710

4842. 195

4849. 7168

4859. 6895
4498. 9401

4499. 9836

4505. 2162

4510. 5259

4521. 221

4530. 3192

4535. 2549

4540. 9988

4545. 9155

4555. 8132

4561. 3481

4570.9717

4588. 4267

4592. 666

4595. 4207

4603. 171

4621. 1621

4631. 7613

4640. 045

4651.55 .5

4663. 2025

4668. 1717

4669. 9843

4673. 6611

4686. 1944

4695. 0380

4703. 9900

4712. 4528

4718. 6162

4729. 1278

4740. 5293

4752. 4141

4761. 1101

4766. 6003

4778. 2940

4789. 3867

4808. 1336

4809. 6140

4822.8549

4826. 7002

4831, 1210

4840. 843

4848. 3623

4858. 3323
4515. 1182 
TABLE 1. Wavelengths of thorium lines-Continued

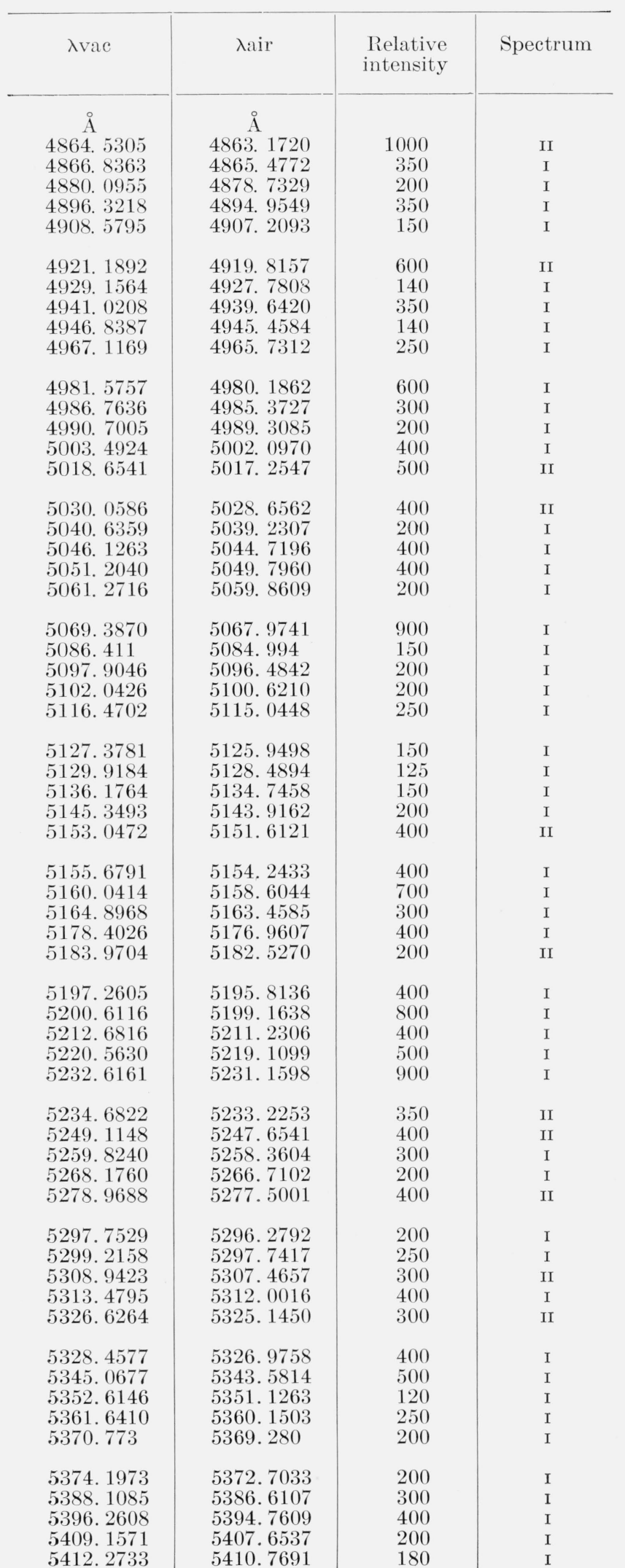

TABLE 1. W avelengths of thorium lines-Continued

\begin{tabular}{|c|c|c|c|}
\hline$\lambda \mathrm{vac}$ & $\lambda$ air & $\begin{array}{l}\text { Relative } \\
\text { intensity }\end{array}$ & Spectrum \\
\hline $\begin{array}{c}\AA \\
5418.9918 \\
5427.1864 \\
5432.6214 \\
5437.4039 \\
5450.9935\end{array}$ & $\begin{array}{c}\AA \\
5417.4858 \\
5425.6782 \\
5431.1118 \\
5435.8930 \\
5449.4790\end{array}$ & $\begin{array}{l}200 \\
250 \\
300 \\
400 \\
150\end{array}$ & $\begin{array}{c}\text { I } \\
\text { II } \\
\text { I } \\
\text { II } \\
\text { II }\end{array}$ \\
\hline $\begin{array}{l}5453.7341 \\
5465.7236 \\
5472.2790 \\
5494.168 \\
5500.7831\end{array}$ & $\begin{array}{l}5452.2188 \\
5464.2051 \\
5470.7588 \\
5492.642 \\
5499.2553\end{array}$ & $\begin{array}{r}250 \\
75 \\
100 \\
100 \\
250\end{array}$ & $\begin{array}{l}\text { I } \\
\text { I } \\
\text { I } \\
\text { I } \\
\text { I }\end{array}$ \\
\hline $\begin{array}{l}5505.8312 \\
5511.5245 \\
5516.4051 \\
5526.1178 \\
5540.8005\end{array}$ & $\begin{array}{l}5504.3020 \\
5509.9938 \\
5514.8731 \\
5524.5832 \\
5539.2620\end{array}$ & $\begin{array}{l}160 \\
300 \\
160 \\
100 \\
400\end{array}$ & $\begin{array}{l}\text { I } \\
\text { I } \\
\text { I } \\
\text { I } \\
\text { I }\end{array}$ \\
\hline $\begin{array}{l}5549.7170 \\
5558.5886 \\
5559.8863 \\
5572.7389 \\
5574.9017\end{array}$ & $\begin{array}{l}5548.1761 \\
5557.0453 \\
5558.3427 \\
5571.1919 \\
5573.3541\end{array}$ & $\begin{array}{l}300 \\
200 \\
400 \\
300 \\
350\end{array}$ & $\begin{array}{l}\text { I } \\
\text { I } \\
\text { I } \\
\text { I } \\
\text { I }\end{array}$ \\
\hline $\begin{array}{l}5580.9078 \\
5588.5781 \\
5596.6168 \\
5603.1587 \\
5613.6262\end{array}$ & $\begin{array}{l}5579.3586 \\
5587.0268 \\
5595.0634 \\
5601.6035 \\
5612.0682\end{array}$ & $\begin{array}{l}300 \\
500 \\
200 \\
150 \\
100\end{array}$ & $\begin{array}{l}\text { I } \\
\text { I } \\
\text { I } \\
\text { I } \\
\text { I }\end{array}$ \\
\hline $\begin{array}{l}5616.8788 \\
5631.8595 \\
5641.3117 \\
5659.4958 \\
5666.7532\end{array}$ & $\begin{array}{l}5615.3200 \\
5630.2967 \\
5639.7463 \\
5657.9256 \\
5665.1810\end{array}$ & $\begin{array}{r}350 \\
60 \\
250 \\
100 \\
140\end{array}$ & $\begin{array}{c}\text { I } \\
\text { I } \\
\text { II } \\
\text { I } \\
\text { I }\end{array}$ \\
\hline $\begin{array}{l}5676.561 \\
5686.7695 \\
5702.4993 \\
5708.6868 \\
5721.2097\end{array}$ & $\begin{array}{l}5674.986 \\
5685.1920 \\
5700.9176 \\
5707.1034 \\
5719.6230\end{array}$ & $\begin{array}{l}120 \\
150 \\
150 \\
150 \\
200\end{array}$ & $\begin{array}{c}\text { I } \\
\text { I } \\
\text { II } \\
\text { II } \\
\text { I }\end{array}$ \\
\hline $\begin{array}{l}5721.7704 \\
5726.9767 \\
5750.3347 \\
5754.6225 \\
5762.1489\end{array}$ & $\begin{array}{l}5720.1835 \\
5725.3884 \\
5748.7402 \\
5753.0268 \\
5760.5512\end{array}$ & $\begin{array}{l}400 \\
250 \\
150 \\
100 \\
600\end{array}$ & $\begin{array}{l}\text { I } \\
\text { I } \\
\text { I } \\
\text { I } \\
\text { I }\end{array}$ \\
\hline $\begin{array}{l}5765.1270 \\
5769.7813 \\
5775.5478 \\
5791.2500 \\
5794.0363\end{array}$ & $\begin{array}{l}5763.5285 \\
5768.1816 \\
5773.9465 \\
5789.6445 \\
5792.4301\end{array}$ & $\begin{array}{r}80 \\
150 \\
150 \\
200 \\
150\end{array}$ & $\begin{array}{l}\text { I } \\
\text { I } \\
\text { I } \\
\text { I } \\
\text { I }\end{array}$ \\
\hline $\begin{array}{l}5797.6759 \\
5802.4380 \\
5805.7507 \\
5814.5842 \\
5817.0346\end{array}$ & $\begin{array}{l}5796.0687 \\
5800.8295 \\
5804.1414 \\
5812.9725 \\
5815.4222\end{array}$ & $\begin{array}{l}150 \\
175 \\
300 \\
150 \\
175\end{array}$ & $\begin{array}{l}\text { I } \\
\text { I } \\
\text { I } \\
\text { I } \\
\text { II }\end{array}$ \\
\hline $\begin{array}{l}5833.9875 \\
5854.3043 \\
5855.7424 \\
5861.2904 \\
5869.9996\end{array}$ & $\begin{array}{l}5832.3706 \\
5852.6820 \\
5854.1197 \\
5859.6662 \\
5868.3731\end{array}$ & $\begin{array}{l}125 \\
200 \\
100 \\
140 \\
125\end{array}$ & $\begin{array}{c}\text { I } \\
\text { I } \\
\text { I } \\
\text { II } \\
\text { I }\end{array}$ \\
\hline $\begin{array}{l}5887.3330 \\
5893.0838 \\
5907.2068 \\
5916.3095 \\
5927.8744\end{array}$ & $\begin{array}{l}5885.7018 \\
5891.4511 \\
5905.5703 \\
5914.6706 \\
5926.2324\end{array}$ & $\begin{array}{r}120 \\
70 \\
100 \\
140 \\
100\end{array}$ & $\begin{array}{l}\text { I } \\
\text { I } \\
\text { I } \\
\text { I } \\
\text { I }\end{array}$ \\
\hline
\end{tabular}


TABLE 1. Wavelengths of thorium lines - Continued

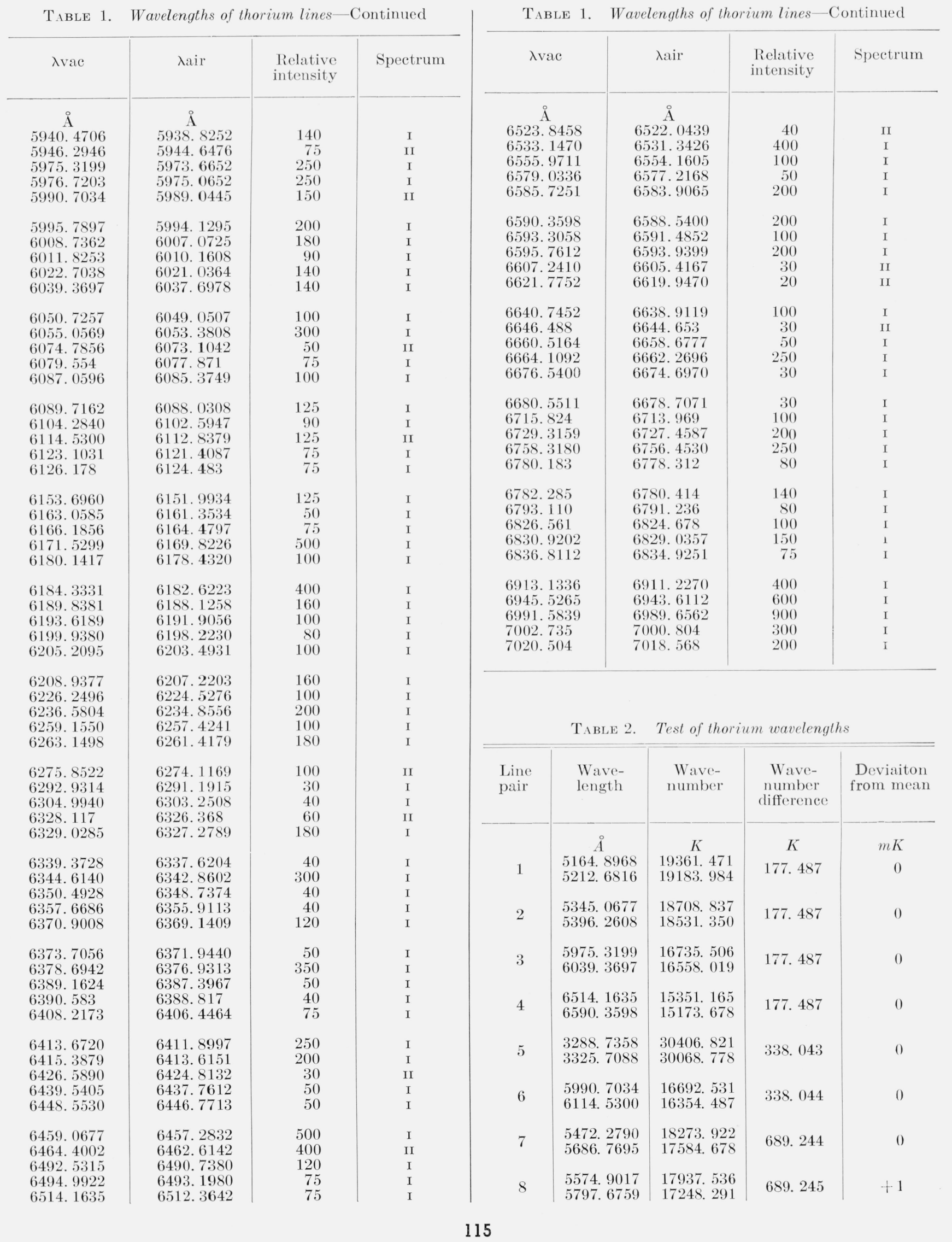

TABLE 1. Wavelengths of thorium lines - Continued

TABLE 2. Test of thorium wavelengths 
TABLE 2. Test of thorium wavelengths-Continued

\begin{tabular}{|c|c|c|c|c|c|c|c|c|c|}
\hline $\begin{array}{l}\text { Line } \\
\text { pair }\end{array}$ & $\begin{array}{l}\text { Wave- } \\
\text { length }\end{array}$ & $\begin{array}{l}\text { Wave- } \\
\text { number }\end{array}$ & $\begin{array}{c}\text { Wave- } \\
\text { number } \\
\text { difference }\end{array}$ & $\begin{array}{c}\text { Deviation } \\
\text { from mean }\end{array}$ & $\begin{array}{l}\text { Line } \\
\text { pair }\end{array}$ & $\begin{array}{l}\text { Wave- } \\
\text { length }\end{array}$ & $\begin{array}{l}\text { Wave- } \\
\text { number }\end{array}$ & $\begin{array}{l}\text { Wave- } \\
\text { number } \\
\text { difference }\end{array}$ & $\begin{array}{l}\text { Deviation } \\
\text { from mean }\end{array}$ \\
\hline 9 & $\begin{array}{c}\stackrel{\AA}{\AA} \\
5769.7813 \\
6008.7362\end{array}$ & $\begin{array}{c}K \\
17331.679 \\
16642.435\end{array}$ & $\begin{array}{c}K \\
689.244\end{array}$ & $\begin{array}{r}m K \\
0\end{array}$ & 32 & $\begin{array}{c}\stackrel{\circ}{\AA} \\
5040.6359 \\
5396.2608\end{array}$ & $\begin{array}{c}K \\
19838.767 \\
18531.350\end{array}$ & $\begin{array}{c}K \\
1307.417\end{array}$ & $\begin{array}{r}m K \\
0\end{array}$ \\
\hline 10 & $\begin{array}{l}3960.4204 \\
4089.8815\end{array}$ & $\begin{array}{l}25249.844 \\
24450.586\end{array}$ & 799. 258 & +3 & 33 & $\begin{array}{l}\text { 5232. } 6161 \\
5616.8788\end{array}$ & $\begin{array}{l}19110.899 \\
17803.482\end{array}$ & 1307. 417 & 0 \\
\hline 11 & $\begin{array}{l}5775.5478 \\
6055.0569\end{array}$ & $\begin{array}{l}\text { 17314. } 375 \\
\text { 16515. } 121\end{array}$ & 799. 254 & -1 & 34 & $\begin{array}{l}\text { 5097. } 9046 \\
5580.9078\end{array}$ & $\begin{array}{l}\text { 19615. } 903 \\
17918.232\end{array}$ & 1697. 671 & +3 \\
\hline 12 & $\begin{array}{l}5940.4706 \\
6236.5804\end{array}$ & $\begin{array}{l}\text { 16833. } 683 \\
16034.428\end{array}$ & 799. 255 & 0 & 35 & $\begin{array}{l}5259.8240 \\
5775.5478\end{array}$ & $\begin{array}{l}\text { 19012. } 043 \\
17314.375\end{array}$ & 1697. 668 & 0 \\
\hline 13 & $\begin{array}{l}6208.9377 \\
6533.1470\end{array}$ & $\begin{array}{l}16105.815 \\
15306.559\end{array}$ & 799. 256 & +1 & 36 & $\begin{array}{l}\text { 5396. } 2608 \\
\text { 5940. } 4706\end{array}$ & $\begin{array}{l}18531.350 \\
16833.683\end{array}$ & 1697. 667 & -1 \\
\hline 14 & $\begin{array}{l}\text { 6373. } 7056 \\
6715.824\end{array}$ & $\begin{array}{l}\text { 15689. } 460 \\
14890.206\end{array}$ & 799. 254 & -1 & 37 & $\begin{array}{l}\text { 5616. } 8788 \\
6208.9377\end{array}$ & $\begin{array}{l}\text { 17803. } 482 \\
16105.815\end{array}$ & 1697. 667 & -1 \\
\hline 15 & $\begin{array}{l}3413.9919 \\
3512.1610\end{array}$ & $\begin{array}{l}\text { 29291. } 224 \\
\text { 28472. } 499\end{array}$ & 818.725 & -3 & 38 & $\begin{array}{l}\text { 6039. } 3697 \\
6729.3159\end{array}$ & $\begin{array}{l}16558.019 \\
14860.352\end{array}$ & 1697. 667 & -1 \\
\hline 16 & $\begin{array}{l}\text { 3519. } 4096 \\
3623.8281\end{array}$ & $\begin{array}{l}28413.857 \\
27595.128\end{array}$ & 818. 729 & +1 & 39 & $\begin{array}{ll}3585 . & 1986 \\
3875 . & 9602\end{array}$ & $\begin{array}{l}27892.458 \\
25800.059\end{array}$ & 2092. 399 & -1 \\
\hline 17 & $\begin{array}{l}\text { 3585. } 1986 \\
\text { 3693. } 6172\end{array}$ & $\begin{array}{l}\text { 27892. } 458 \\
27073.731\end{array}$ & 818.727 & -1 & 40 & $\begin{array}{l}3728.9626 \\
4044.5369\end{array}$ & $\begin{array}{l}26817.110 \\
24724.710\end{array}$ & 2092. 400 & 0 \\
\hline 18 & $\begin{array}{l}4379.4071 \\
4542.2720\end{array}$ & $\begin{array}{l}22834.141 \\
22015.414\end{array}$ & 818. 727 & -1 & 41 & $\begin{array}{l}\text { 4098. } 9042 \\
4483.4270\end{array}$ & $\begin{array}{l}24396.764 \\
22304.367\end{array}$ & 2092. 397 & -3 \\
\hline 19 & $\begin{array}{ll}4664 . & 5080 \\
4849 . & 7168\end{array}$ & $\begin{array}{l}21438.488 \\
20619.761\end{array}$ & 818.727 & -1 & 42 & $\begin{array}{l}4687.5060 \\
5197.2605\end{array}$ & $\begin{array}{l}21333.306 \\
19240906\end{array}$ & 2092. 400 & 0 \\
\hline 20 & $\begin{array}{l}5136.1764 \\
5361.6410\end{array}$ & $\begin{array}{l}\text { 19469. } 736 \\
18651.006\end{array}$ & 818. 730 & +2 & 43 & $\begin{array}{l}4842.1950 \\
5388.1085\end{array}$ & $\begin{array}{l}\text { 20651. } 791 \\
18559.389\end{array}$ & 2092. 402 & +2 \\
\hline 21 & $\begin{array}{l}5465.7236 \\
5721.7704\end{array}$ & $\begin{array}{l}18295.839 \\
17477.108\end{array}$ & 818. 731 & +3 & 44 & $\begin{array}{l}5003.4924 \\
5588.5781\end{array}$ & $\begin{array}{l}19986.040 \\
17893.639\end{array}$ & 2092. 401 & +1 \\
\hline 22 & $\begin{array}{l}5765.1270 \\
6050.7257\end{array}$ & $\begin{array}{l}17345.672 \\
16526.943\end{array}$ & 818. 729 & +1 & 45 & $\begin{array}{l}5136.1764 \\
5754.6225\end{array}$ & $\begin{array}{l}\text { 19469. } 736 \\
17377.334\end{array}$ & 2092. 402 & +2 \\
\hline 23 & $\begin{array}{l}6184.3331 \\
6514.1635\end{array}$ & $\begin{array}{l}16169.892 \\
15351.165\end{array}$ & 818. 727 & -1 & 46 & $\begin{array}{l}5465.7236 \\
6171.5299\end{array}$ & $\begin{array}{l}18295.839 \\
16203.438\end{array}$ & 2092. 401 & +1 \\
\hline 24 & $\begin{array}{l}5558.5886 \\
5887.3330\end{array}$ & $\begin{array}{l}17990.178 \\
16985.620\end{array}$ & 1004.558 & +1 & 47 & $\begin{array}{l}5765.1270 \\
6555.9711\end{array}$ & $\begin{array}{l}17345.672 \\
15253.270\end{array}$ & 2092. 402 & +2 \\
\hline 25 & $\begin{array}{l}6492.5315 \\
6945.5265\end{array}$ & $\begin{array}{l}15402.313 \\
14397.757\end{array}$ & 1004.556 & -1 & 48 & $\begin{array}{l}3691.6742 \\
4076.6536\end{array}$ & $\begin{array}{l}27087.980 \\
24529.924\end{array}$ & 2558. 056 & -1 \\
\hline 26 & $\begin{array}{l}5432.6214 \\
5791.2500\end{array}$ & $\begin{array}{l}18407.320 \\
17267.429\end{array}$ & 1139. 891 & -2 & 49 & $\begin{array}{l}\text { 3804. } 1547 \\
\text { 4214. } 2542\end{array}$ & $\begin{array}{l}26287.049 \\
23728.991\end{array}$ & 2558. 058 & +1 \\
\hline 27 & $\begin{array}{l}5750.3347 \\
6153.6960\end{array}$ & $\begin{array}{l}17390.292 \\
16250.397\end{array}$ & 1139. 895 & +2 & 50 & $\begin{array}{l}5762.1489 \\
6758.3180\end{array}$ & $\begin{array}{l}17354.637 \\
14796.581\end{array}$ & 2558. 056 & -1 \\
\hline 28 & $\begin{array}{l}5976.7203 \\
6413.6720\end{array}$ & $\begin{array}{l}\text { 16731. } 584 \\
15591.692\end{array}$ & 1139. 892 & -1 & 51 & $\begin{array}{l}3592.4771 \\
3974.3200\end{array}$ & $\begin{array}{l}27835.946 \\
25161.537\end{array}$ & 2674. 409 & 0 \\
\hline 29 & $\begin{array}{l}\text { 6193. } 6189 \\
6664.1092\end{array}$ & $\begin{array}{l}16145.650 \\
15005.757\end{array}$ & 1139. 893 & 0 & 52 & $\begin{array}{l}3764.0030 \\
4185.3170\end{array}$ & $\begin{array}{l}26567.460 \\
23893.053\end{array}$ & 2674.407 & -2 \\
\hline 30 & $\begin{array}{l}4488.1563 \\
4767.9332\end{array}$ & $\begin{array}{l}22280.864 \\
20973.448\end{array}$ & 1307.416 & -1 & 53 & $\begin{array}{l}4828.0490 \\
5549.7170\end{array}$ & $\begin{array}{l}20693.347 \\
18018.937\end{array}$ & 2674.410 & +1 \\
\hline 31 & $\begin{array}{l}4880.0955 \\
5212.6816\end{array}$ & $\begin{array}{l}\text { 20491. } 402 \\
\text { 19183. } 984\end{array}$ & 1307. 418 & +1 & 54 & $\begin{array}{l}5313.4795 \\
6193.6189\end{array}$ & $\begin{array}{l}18820.059 \\
16145.650\end{array}$ & 2674. 409 & 0 \\
\hline
\end{tabular}

TABLE 2. Test of thorium wavelengths-Continued 
TABLE 2. Test of thorium wavelengths-Continued

\begin{tabular}{|c|c|c|}
\hline $\begin{array}{l}\text { Line } \\
\text { pair }\end{array}$ & $\begin{array}{l}\text { Wave- } \\
\text { length }\end{array}$ & $\begin{array}{l}\text { Wave- } \\
\text { number }\end{array}$ \\
\hline 55 & $\begin{array}{c}\stackrel{\AA}{A} \\
5345.0677 \\
6236.5804\end{array}$ & $\begin{array}{c}K \\
18708.837 \\
16034.428\end{array}$ \\
\hline 56 & $\begin{array}{l}5361.6410 \\
6259.1550\end{array}$ & $\begin{array}{l}18651.006 \\
15976.597\end{array}$ \\
\hline 57 & $\begin{array}{l}3310.3178 \\
3657.7354\end{array}$ & $\begin{array}{l}30208.580 \\
27339.320\end{array}$ \\
\hline 58 & $\begin{array}{l}3331.4345 \\
3683.5345\end{array}$ & $\begin{array}{l}30017.099 \\
27147.839\end{array}$ \\
\hline 59 & $\begin{array}{l}3458.0588 \\
3838.9638\end{array}$ & $\begin{array}{l}28917.958 \\
26048.696\end{array}$ \\
\hline 60 & $\begin{array}{l}3599.1464 \\
4013.6295\end{array}$ & $\begin{array}{l}27784.366 \\
24915.105\end{array}$ \\
\hline 61 & $\begin{array}{l}3643.2867 \\
4068.5994\end{array}$ & $\begin{array}{l}27447.744 \\
24578.483\end{array}$ \\
\hline 62 & $\begin{array}{l}3840.7835 \\
4316.4680\end{array}$ & $\begin{array}{l}26036.354 \\
23167.090\end{array}$ \\
\hline 63 & $\begin{array}{l}4113.9150 \\
4664.5080\end{array}$ & $\begin{array}{l}24307.746 \\
21438.488\end{array}$ \\
\hline 64 & $\begin{array}{l}4251.5110 \\
4842.195\end{array}$ & $\begin{array}{l}23521.049 \\
20651.791\end{array}$ \\
\hline 65 & $\begin{array}{l}4375.3536 \\
5003.4924\end{array}$ & $\begin{array}{l}22855.296 \\
19986.040\end{array}$ \\
\hline 66 & $\begin{array}{l}4494.5943 \\
5160.0414\end{array}$ & $\begin{array}{l}22248.949 \\
19379.689\end{array}$ \\
\hline 67 & $\begin{array}{l}4946.8387 \\
5765.1270\end{array}$ & $\begin{array}{l}20214.930 \\
17345.672\end{array}$ \\
\hline 68 & $\begin{array}{l}5127.3781 \\
6011.8253\end{array}$ & $\begin{array}{l}19503.145 \\
16633.883\end{array}$ \\
\hline 69 & $\begin{array}{l}5603.1587 \\
6676.5400\end{array}$ & $\begin{array}{l}17847.076 \\
14977.818\end{array}$ \\
\hline 70 & $\begin{array}{l}4483.4270 \\
5220.5630\end{array}$ & $\begin{array}{l}22304.367 \\
19155.022\end{array}$ \\
\hline 71 & $\begin{array}{l}5588.5781 \\
6782.285\end{array}$ & $\begin{array}{l}\text { 17893. } 639 \\
14744.293\end{array}$ \\
\hline 72 & $\begin{array}{l}3512.1610 \\
4019.2344\end{array}$ & $\begin{array}{l}28472.499 \\
24880.360\end{array}$ \\
\hline 73 & $\begin{array}{l}3920.1336 \\
4562.6266\end{array}$ & $\begin{array}{l}\text { 25509. } 335 \\
21917.200\end{array}$ \\
\hline 74 & $\begin{array}{l}\text { 4596. } 7082 \\
5505.8312\end{array}$ & $\begin{array}{l}21754.698 \\
18162.562\end{array}$ \\
\hline 75 & $\begin{array}{l}3305.1895 \\
3764.0030\end{array}$ & $\begin{array}{l}\text { 30255. } 451 \\
26567.460\end{array}$ \\
\hline 76 & $\begin{array}{l}\text { 3424. } 9714 \\
\text { 3920. } 1336\end{array}$ & $\begin{array}{l}\text { 29197. } 324 \\
25509.335\end{array}$ \\
\hline 77 & $\begin{array}{l}3829.4708 \\
4459.2531\end{array}$ & $\begin{array}{l}\text { 26113. } 269 \\
22425.280\end{array}$ \\
\hline
\end{tabular}

TABLE 2. Test of thorium wavelengths-Continued

\begin{tabular}{|c|c|c|c|c|}
\hline $\begin{array}{l}\text { Line } \\
\text { pair }\end{array}$ & $\begin{array}{l}\text { Wave- } \\
\text { length }\end{array}$ & $\begin{array}{l}\text { Wave- } \\
\text { number }\end{array}$ & $\begin{array}{c}\text { Wave- } \\
\text { number } \\
\text { difference }\end{array}$ & $\begin{array}{l}\text { Deviation } \\
\text { from mean }\end{array}$ \\
\hline 78 & $\begin{array}{c}\stackrel{\AA}{A} \\
3831.8604 \\
4462.4933\end{array}$ & $\begin{array}{c}K \\
26096.984 \\
22408.997\end{array}$ & $\begin{array}{c}K \\
3687.987\end{array}$ & $\begin{array}{r}m K \\
0\end{array}$ \\
\hline 79 & $\begin{array}{l}4003.0253 \\
4696.3520\end{array}$ & $\begin{array}{l}24981.106 \\
21293.122\end{array}$ & 3687.984 & -3 \\
\hline 80 & $\begin{array}{l}4101.4985 \\
4832.4710\end{array}$ & $\begin{array}{l}\text { 24381. } 333 \\
\text { 20693. } 347\end{array}$ & 3687.986 & -1 \\
\hline 81 & $\begin{array}{l}4113.9150 \\
4849.7168\end{array}$ & $\begin{array}{l}24307.746 \\
20619.761\end{array}$ & 3687.985 & -2 \\
\hline 82 & $\begin{array}{l}4212.1096 \\
4986.7636\end{array}$ & $\begin{array}{l}23741.073 \\
20053.086\end{array}$ & 3687.987 & 0 \\
\hline 83 & $\begin{array}{l}4338.4971 \\
5164.8968\end{array}$ & $\begin{array}{l}23049.456 \\
\text { 19361. } 471\end{array}$ & 3687.985 & -2 \\
\hline 84 & $\begin{array}{l}4516.3845 \\
5418.9918\end{array}$ & $\begin{array}{l}22141.605 \\
18453.617\end{array}$ & 3687.988 & +1 \\
\hline 85 & $\begin{array}{l}4779.6300 \\
5802.4380\end{array}$ & $\begin{array}{l}20922.122 \\
17234.135\end{array}$ & 3687. 987 & 0 \\
\hline 86 & $\begin{array}{l}\text { 4896. } 3218 \\
5975.3199\end{array}$ & $\begin{array}{l}20423.494 \\
16735.506\end{array}$ & 3687.988 & +1 \\
\hline 87 & $\begin{array}{l}4946.8387 \\
6050.7257\end{array}$ & $\begin{array}{l}20214.930 \\
16526.940\end{array}$ & 3687.990 & +3 \\
\hline 88 & $\begin{array}{l}5046.1263 \\
6199.9380\end{array}$ & $\begin{array}{l}19817.181 \\
16129.194\end{array}$ & 3687.987 & 0 \\
\hline 89 & $\begin{array}{l}3458.0588 \\
3991.6206\end{array}$ & $\begin{array}{l}28917.958 \\
25052.481\end{array}$ & 3865.477 & +4 \\
\hline 90 & $\begin{array}{l}4258.6948 \\
5097.9046\end{array}$ & $\begin{array}{l}23481.373 \\
19615.903\end{array}$ & 3865.470 & -3 \\
\hline 91 & $\begin{array}{l}4330.1329 \\
5200.6116\end{array}$ & $\begin{array}{l}23093.979 \\
19228.508\end{array}$ & 3865.471 & -2 \\
\hline 92 & $\begin{array}{l}4338.4971 \\
5212.6816\end{array}$ & $\begin{array}{l}23049.456 \\
19183.984\end{array}$ & 3865.472 & -1 \\
\hline 93 & $\begin{array}{l}4371.1038 \\
5259.8240\end{array}$ & $\begin{array}{l}22877.517 \\
19012.043\end{array}$ & 3865.474 & +1 \\
\hline 94 & $\begin{array}{l}\text { 4896. } 3218 \\
6039.3697\end{array}$ & $\begin{array}{l}20423.494 \\
16558.019\end{array}$ & 3865.475 & +2 \\
\hline 95 & $\begin{array}{l}3532.4595 \\
4261.5321\end{array}$ & $\begin{array}{l}28308.888 \\
23465.739\end{array}$ & 4843.149 & +1 \\
\hline 96 & $\begin{array}{l}4216.0158 \\
5297.7529\end{array}$ & $\begin{array}{l}23719.076 \\
18875.928\end{array}$ & 4843.148 & 0 \\
\hline 97 & $\begin{array}{l}4350.2950 \\
5511.5245\end{array}$ & $\begin{array}{l}22986.947 \\
18143.800\end{array}$ & 4843.147 & -1 \\
\hline 98 & $\begin{array}{l}3381.8300 \\
4110.4832\end{array}$ & $\begin{array}{l}29569.789 \\
24328.040\end{array}$ & 5241.749 & 0 \\
\hline 99 & $\begin{array}{l}3438.2923 \\
4194.1984\end{array}$ & $\begin{array}{l}29084.206 \\
23842.458\end{array}$ & 5241.748 & 0 \\
\hline 100 & $\begin{array}{l}3349.7310 \\
4116.9203\end{array}$ & $\begin{array}{l}29853.143 \\
24290.001\end{array}$ & 5563.142 & 0 \\
\hline
\end{tabular}


TABLE 2. Test of thorium wavelengths-Continued

\begin{tabular}{|c|c|c|c|c|}
\hline $\begin{array}{l}\text { Line } \\
\text { pair }\end{array}$ & $\begin{array}{l}\text { Wave- } \\
\text { length }\end{array}$ & $\begin{array}{l}\text { Wave- } \\
\text { number }\end{array}$ & $\begin{array}{c}\text { Wave- } \\
\text { number } \\
\text { difference }\end{array}$ & $\begin{array}{l}\text { Deviation } \\
\text { from mean }\end{array}$ \\
\hline 101 & $\begin{array}{c}\stackrel{\AA}{A} \\
3599.1464 \\
4500.2022\end{array}$ & $\begin{array}{c}K \\
27784.366 \\
22221.224\end{array}$ & $\begin{array}{c}K \\
5563.142\end{array}$ & $\begin{array}{r}m K \\
0\end{array}$ \\
\hline 102 & $\begin{array}{l}4258.6948 \\
5580.9078\end{array}$ & $\begin{array}{l}23481.373 \\
17918.232\end{array}$ & 5563.141 & +1 \\
\hline 103 & $\begin{array}{l}4371.1038 \\
5775.5478\end{array}$ & $\begin{array}{l}22877.517 \\
17314.375\end{array}$ & 5563.142 & 0 \\
\hline 104 & $\begin{array}{l}4705.3063 \\
6373.7056\end{array}$ & $\begin{array}{l}21252.602 \\
15689.460\end{array}$ & 5563.142 & 0 \\
\hline 105 & $\begin{array}{l}4896.3218 \\
6729.3159\end{array}$ & $\begin{array}{l}20423.494 \\
14860.352\end{array}$ & 5563.142 & 0 \\
\hline 106 & $\begin{array}{l}3305.1895 \\
4185.3170\end{array}$ & $\begin{array}{l}30255.451 \\
23893.053\end{array}$ & 6362.398 & +2 \\
\hline 107 & $\begin{array}{l}4037.1879 \\
5432.6214\end{array}$ & $\begin{array}{l}24769.717 \\
18407.320\end{array}$ & 6362.397 & +1 \\
\hline 108 & $\begin{array}{l}4101.4985 \\
5549.7170\end{array}$ & $\begin{array}{l}24381.333 \\
18018.937\end{array}$ & 6362.396 & 0 \\
\hline 109 & $\begin{array}{l}4330.1329 \\
5976.7203\end{array}$ & $\begin{array}{l}23093.979 \\
16731.584\end{array}$ & 6362.395 & -1 \\
\hline 110 & $\begin{array}{l}4371.1038 \\
6055.0569\end{array}$ & $\begin{array}{l}22877.517 \\
16515.121\end{array}$ & 6362.396 & 0 \\
\hline 111 & $\begin{array}{l}4705.3063 \\
6715.824\end{array}$ & $\begin{array}{l}21252.602 \\
14890.206\end{array}$ & 6362.396 & 0 \\
\hline
\end{tabular}

\section{References}

[1] W. F. Meggers, Trans. I.A.U. 9, 225-226 (1955).

[2] W. F. Meggers and R. W. Stanley, J. Res. NBS 61, 95-103 (1958).

[3] K. G. Kessler and W. F. Meggers, J. Opt. Soc. Am. 45, 902 (1955).

[4] F. H. Rolt and H. Barrell, Proc. Roy. Soc. A 122, 131-133 (1929).

[5] V. Kaufman, J. Opt. Soc. Am. 52, 866-870 (1962).

[6] C. D. Coleman, W. R. Bozman and W. F. Meggers, Table of Wavenumbers, 2000 A to 7000 A, NBS Monograph 3, Vol. 1, 500 pp. (1960).

[7] R. Zalubas, NBS Monograph 17,103 pp. (1960).

[8] R. Zalubas, J. Res. NBS 63A, 275-278 (1959).

[9] G. W. Charles, Compilation of Data on Some Spectra of Thorium, ORNL 2319, May 8, 1958, $162 \mathrm{pp}$.

[10] T. A. Littlefield, Trans. I.A.U. 11 B, 212 (1961).

[11] R. W. Stanley and W. F. Meggers, J. Res. NBS 58, 41-49 (1957)

[12] T. A. Littlefield and W. A. Wood, Trans. I.A.U. 11A, 105-108 (1961). I.A.U. Agenda and Draft Reports, p. 142 (1964).

[13] A. Davison, A. Giacchetti and R. W. Stanley, J. Opt. Soc. Am. 52, 447-451 (1962).

[14] A. Giacchetti, M. Gallardo, M. J. Garavaglia, Z. Gonzalez, F. P. J. Valero, and E. Zakowicz, J. Opt. Soc. Am. 54, 957-959 (1964). 